\title{
Neural Network For Modeling Nonlinear Time Series: A New Approach
}

\author{
Chokri Slim ${ }^{1}$ \\ Abdelwahed Trabelsi ${ }^{2}$ \\ ${ }^{1}$ University of Tunis, Bestmod Laboratory, 41 rue de la liberté \\ 2000 Le Bardo, Tunisia \\ chokri.slim@iscae.rnu.tn \\ ${ }^{2}$ University of Tunis, Bestmod Laboratory, 41rue de la liberté \\ 2000 Le Bardo, Tunisia \\ abdel.trabelsi@isg.rnu.tn
}

\begin{abstract}
Nonlinear modeling with neural networks offers a promising approach for studying the prediction of a chaotic time series. In this paper, we propose a neural net based on Extended Kalman Filter to examine the nonlinear dynamic proprieties of some financial time series in order to differentiate between low-dimensional chaos and stochastic behavior. Kalman filtering, because it can deal with varying unobservable states, provides an efficient framework to model these non-stationary exposures. A controlled simulation experiment is used to introduce the issues involved and to present the proposed approach. Measures of forecast accuracy are developed. The pertinence of this model is discussed from the Tunisian Stock Exchange database.
\end{abstract}

\section{Introduction}

Within the past decades, there has been a growing interest in applying nonlinear models to predict chaotic time series [1]. The major problem in these researches is the difficulty of distinguishing between deterministic chaos and purely random processes. An appreciable amount of recent literature deals with this subject [2] and [3].

By new neural network architecture we allow additional information flows between the different outputs and as a sequence we get a better representation of the underlying dynamic system. The net is based on multilayer feed forward architecture with random connections. The model can then convert to its equivalent state space representation. Using this state space form, a Bayesian inferential algorithm based on non linear Kalman Filter is derived to estimate the state. We analyze the net of a chaotic time series (logistic map) by using the dynamic invariant that characterizes the attractor, the largest Lyaponov exponent. This approach permits us to claim that the net capture "chaos" if it learns the dynamic invariant of a chaotic time series. A detailed step by step description of the methodology is presented to facilitate the use of this new method. Some issues related to the practical use of the proposed model in the context of the Tunisian Financial market are also considered.

P.M.A. Sloot et al. (Eds.): ICCS 2003, LNCS 2659, pp. 159-168, 2003.

(C) Springer-Verlag Berlin Heidelberg 2003 


\section{Neural Net Architecture}

A general form of nonlinear time series with functional coefficients can be given by:

$$
Y_{\mathrm{t}}=f\left(\mathbf{X}_{\mathrm{t}}, \varphi\left(\mathbf{X}_{\mathrm{t}}\right), \gamma\left(\mathbf{X}_{\mathrm{t}}\right)\right)+\xi_{\mathrm{t}}
$$

Where $f($.$) : is unknown non linear function. Y_{\mathrm{t}}$ : the time series.

$\mathbf{X}_{\mathrm{t}}=\left(Y_{t-1}, Y_{t-2}, \ldots, Y_{t-p}, Z_{t-d-1}, Z_{t-d-2}, \ldots, Z_{t-d-m}\right):$ is a vector of $p$-past values time series $Y_{t}$ and $m$-past values input $Z_{t}$. This vector reflect some changing environmental conditions that causes the system's parameters to vary; $p, m, d$ : represent the orders and time delay of the mode; $Z_{t}$ : the measured input; $\varphi\left(\mathbf{X}_{t}\right)$ and $\gamma\left(\mathbf{X}_{t}\right)$ : are vectors of unknown parameters which are functions of $\mathbf{X}_{t} ; \xi_{t}$ : is a sequence of iid random variables with mean 0 and variance $R_{t}$.

The main difficulty in using the proposed model in (1) is specifying the functional coefficients. We consider in this section, a special class of neural networks called Tangent Hyperbolic neural Networks THNN to identify the model in (1). The most important feature of the THNN is the smooth output which is due to the shape of the tangent hyperbolic functions. It has been proved that this class of networks can approximate continuous functions at any arbitrary accuracy. A complete description of a Neural Networks theory and the application of neural networks to the problem of nonlinear system identification and prediction can be found in [5], [6]. The non linear coefficients $\varphi_{i}\left(\mathbf{X}_{\mathrm{t}}\right)$ and $\gamma_{j}\left(\mathbf{X}_{\mathrm{t}}\right)$ in (1) can be approximated by a sub-THNN, then:

$$
\begin{aligned}
\hat{\varphi_{i}}\left(\mathbf{X}_{t}\right) & =\sum_{k=1}^{q} w_{k}^{\varphi_{i}} h_{k}\left(\mathbf{X}_{t}\right) . \\
\gamma_{j}\left(\mathbf{X}_{t}\right) & =\sum_{k=1}^{q} w_{k}^{\gamma_{j}} h_{k}\left(\mathbf{X}_{t}\right) .
\end{aligned}
$$

Representing each parameter of (2) and (3) as a neural network in figure1, the overall estimate model (1) can be represented in figure1, which is a two layered THNN structure. The first layer is composed of sub networks and the second layer is the output given by:

$$
Y_{t}=h\left(\mathbf{X}_{t}, \hat{\varphi}\left(\mathbf{X}_{t}\right), \hat{\gamma}\left(\mathbf{X}_{t}\right)\right)+\xi
$$

We call this new neural network a stochastic neural net (SNN). We propose, now, a Gradient Back propagation algorithm to train the free parameters which are the weights of the sub networks in the first layer of the SNN, while the "weights" of the second layer are viewed as fixed in every iteration of this algorithm. Denote:

$$
\begin{gathered}
\beta_{t}^{\varphi}=\left\lfloor w_{1}^{\varphi}, w_{2}^{\varphi_{i}, \ldots, w_{q}^{\varphi}} \varphi_{i}\right\rfloor \in I R^{q}, i=1, \ldots, m . \\
\beta_{t}^{\gamma_{j}}=\left\lfloor w_{1}^{\gamma_{j}}, w_{2}^{\gamma_{j}}, \ldots w_{q}^{\gamma_{j}}\right\rfloor \in I R^{q}, j=1, \ldots, m .
\end{gathered}
$$




$$
\begin{aligned}
& \mathbf{B}_{t-1}=\left[\beta_{t}^{\varphi_{1}} \beta_{t}^{\varphi_{2}} \ldots \beta_{t}^{\varphi_{p}} \beta_{t}^{\gamma_{1}} \beta_{t}^{\gamma_{2}} \ldots \beta_{t}^{\gamma_{m}}\right]^{\mathrm{T}} \in I R^{q x(p+m)} \\
& \mathbf{h}_{t-1}=\left[h_{1}\left(\mathrm{X}_{\mathrm{t}}\right), h_{2}\left(\mathrm{X}_{\mathrm{t}}\right), \ldots, h_{q}\left(\mathrm{X}_{\mathrm{t}}\right)\right]^{\mathrm{T}} \in I R^{\mathrm{q}} . \\
& \mathbf{F}_{t-1}=\left[Y_{t-1}, Y_{t-2}, \ldots, Y_{t-p}, Z_{t-d-1}, \ldots, Z_{t-d-m}\right]^{\mathrm{T}} \in I R \\
& \mathbf{O}_{t-1}=\left[\varphi_{1}\left(\mathbf{X}_{t}\right), \ldots, \varphi_{p}\left(\mathbf{X}_{t}\right), \gamma_{1}\left(\mathbf{X}_{t}\right), \ldots, \gamma_{m}\left(\mathbf{X}_{t}\right)\right] \in I R^{\mathrm{p}+\mathrm{m}}
\end{aligned}
$$

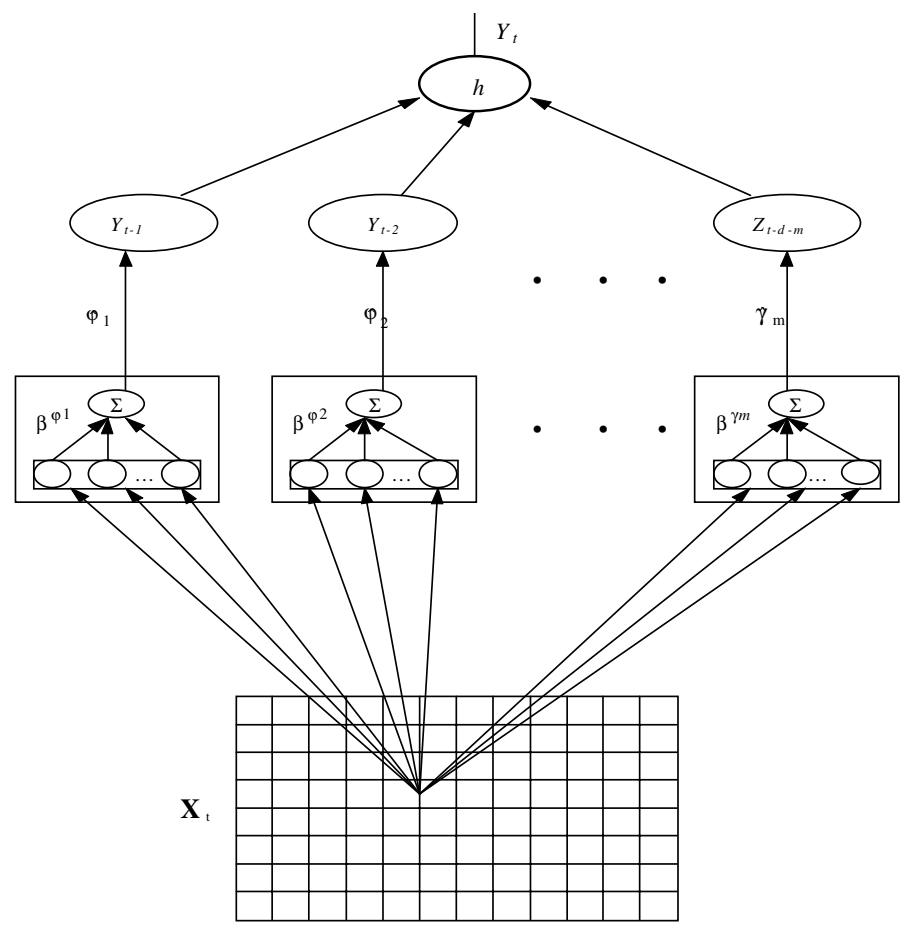

Fig. 1. A stochastic neural network for non linear time series

A time t, the input vector $\mathbf{F}_{\mathrm{t}-1}$ is presented to the neural network shown in figure1. In the forward pass the networks calculates the output according to (2) and (3). The output of the first layer and final output of the SNN are given by:

$$
\begin{gathered}
\mathbf{O}_{t-1}=\mathbf{h}_{t-1} \mathbf{B}_{t-1} . \\
\hat{Y}_{t}=h\left(\mathbf{O}_{t-1} \mathbf{F}_{t-1}\right)=h\left(\mathbf{h}_{t-1}^{\mathrm{T}} \mathbf{B}_{t-1} \mathbf{F}_{t-1}\right) .
\end{gathered}
$$

The error function to be minimized is: 


$$
J=\frac{1}{2} \sum_{t-1}^{n}\left(Y_{t}-\hat{Y}_{t}\right)^{2}
$$

The algorithm computes the instantaneous gradient of the network's error function $(J)$ :

$$
\hat{\nabla}_{t-1}=\frac{\partial J}{\partial \mathbf{B}_{t-1}} .
$$

And the updates the weights according to the steepest descent equation:

$$
\mathbf{B}_{t}=\mathbf{B}_{t-1}-\mu \hat{\nabla}_{t-1}
$$

Where $\mu$ is the learning rate. Let:

$$
\varepsilon_{t}=Y_{t}-\hat{Y}_{t}
$$

$h($.$) : is the tangent hyperbolic function.$

We can now perform the steepest descent procedure to each weight in the second layer:

$$
\begin{array}{cc}
\beta_{t}^{\varphi_{i}}=\beta_{t-1}^{\varphi_{i}}+\mu \varepsilon_{t} Y_{t-i}\left(1-\hat{Y}_{t}^{2}\right) \mathbf{h}_{t-1}^{\mathrm{T}} & \text { for } i=1,2, \ldots, p \\
\beta_{t}^{\gamma_{j}{ }^{\prime}}=\beta_{t-1}^{\gamma_{j}}+\mu \varepsilon_{t} Z_{t-d-j}\left(1-\hat{Y}_{t}^{2}\right) \mathbf{h}_{t-1}^{\mathrm{T}} & \text { for } j=1,2, \ldots, m .
\end{array}
$$

This completes the description of the gradient back propagation algorithm and the next section discusses the corresponding non linear state space method.

\section{Nonlinear State Space Representation}

Having identified the system model using the above neural network algorithm, it is useful to recast the SNN in the state-space form to perform state estimation. This requires the coefficients $\varphi_{i}\left(\mathbf{X}_{t}\right)$ and $\gamma_{j}\left(\mathbf{X}_{t}\right)$ as function of time. In this case the SNN can be in the following form:

$$
Y_{t}=h\left(\sum_{i=1}^{p} \varphi_{i, t} Y_{t-i}+\sum_{j=1}^{m} \gamma_{j, t} Z_{t-d-j}\right) .
$$

The non linear state space of $\mathbf{S N N}$ is given by:

$$
\begin{gathered}
\Theta_{t+1}=\mathbf{H}\left(\Theta_{t}, \varphi_{t}, \bar{Z}_{t}\right)+\omega_{t} . \\
Y_{t}=h\left(\Theta_{t}, \bar{Z}_{t}\right)+\xi_{t} .
\end{gathered}
$$


$\Theta_{t}=\left(\begin{array}{c}\theta_{1, t} \\ \theta_{2, t} \\ \ldots \\ \theta_{p-1, t} \\ \theta_{p, t}\end{array}\right) ; \quad \mathbf{H}\left(\Theta_{t}, \varphi_{t}, \bar{Z}_{t}\right)=\left(\begin{array}{c}Y_{t-p+1}=\theta_{2, t} \\ Y_{t-p+2}=\theta_{3, t} \\ \ldots \\ Y_{t-1}=\theta_{p, t} \\ Y_{t}=h\left(\varphi_{1, t} \theta_{p, t}+\ldots+\varphi_{p, t} \theta_{1, t}\right.\end{array}\right)$

And $: \bar{Z}_{t}=\sum_{\mathrm{j}=1}^{\mathrm{m}} \gamma_{j, t} Z_{t-d-j}$

Equations (20) and (21) represent respectively the transition equation and the observation equation, with $\Theta_{t}$ is the state vector of the system at time $\mathrm{t}$ and $Y_{t}$ is the observation vector at time t. The $\omega_{t}$ is of innovations, with zero mean and variance $\mathrm{Q}_{t}$, $\xi_{t}$ is an additive measurement noise with zero mean and variance $\mathrm{R}_{t}$. We assume that the noise vectors are uncorrelated with covariance: $P^{\omega \xi}=\left(\begin{array}{cc}Q_{t} & 0 \\ 0 & R_{t}\end{array}\right)$.

\section{Bayesian Inferential Algorithm Based on Extended Kalman Filter}

The Kalman filer is a set of mathematical equations that provides an efficient computational (recursive) solution of the least-squares method. The filter is very powerful in several aspects: it supports estimations of past, present, and even future states, and it can do so even when the precise nature of modeled system is unknown. A complete discussion to the idea of the Kalman filter can be found in [7].

With the above non linear state space model of (20), the application of the Kalman filter is not straightforward because the model is not linear. We are established, a recursive algorithm for the measurement and the state update for the dynamic system represented by the SNN. This algorithm can be summarized by the update recursion equations.

$$
\begin{gathered}
\hat{\Theta}_{\mathrm{t}+1 / \mathrm{t}}=H\left[\left(\hat{\Theta}_{\mathrm{t} / \mathrm{t}}, \bar{Z}_{t}\right)\right] \\
P_{t+1 / t}^{\Theta}=\mathbf{A}_{t} P_{t / t}^{\Theta} \mathbf{A}_{t}^{\mathrm{T}}+Q_{\mathrm{t}} . \\
K_{t+1}=P_{t+1 / t}^{\Theta} \mathbf{G}_{t+1}^{\mathrm{T}}\left(\mathbf{G}_{t+1} P_{t+1 / t}^{\Theta} \mathbf{G}_{t+1}^{\mathrm{T}}+R_{\mathrm{t}+1}\right)^{-1} . \\
\hat{\Theta}_{t+1 / t+1}=\hat{\Theta}_{t+1 / t}+K_{t+1}\left(Y_{t+1}-h\left(\hat{\Theta}_{t+1 / t}, \overline{\mathrm{Z}}_{\mathrm{t}+1}\right)\right) . \\
P_{t+1 / t+1}^{\Theta}=P_{t+1 / t}^{\Theta}\left(\mathbf{I}-K_{t+1} \mathbf{G}_{t+1}\right) . \\
\left.\hat{Y}_{t+1 / t}=h\left(\hat{\Theta}_{t+1 / t}, \overline{\mathrm{Z}}_{\mathrm{t}+1}\right)\right) .
\end{gathered}
$$


Where $P_{t+1 / t}^{\Theta}$ : the prior estimate covariance of the state;

$K_{t}$ : the gain filter $\mathbf{A}_{\mathrm{t}}=\frac{\partial H\left[\left(\hat{\Theta}_{\mathrm{t} / \mathrm{t}}, \bar{Z}_{t}\right)\right]}{\partial\left(\hat{\Theta}_{\mathrm{t} / \mathrm{t}}\right)} ; G_{\mathrm{t}+1}=\frac{\partial h\left(\hat{\Theta}_{\mathrm{t}+1 / \mathrm{t}}, \bar{Z}_{t+1}\right)}{\partial\left(\hat{\Theta}_{\mathrm{t}+1 / \mathrm{t}}\right)}$.

Equations (22) and (23) are the time update equations, these equations are responsible for projecting forward (in time) the current state and error covariance estimates to obtain the prior estimates for the next time step. Equations (24) to (27) are the measurement update equations, the first task during the measurement update is to compute the Kalman gain, $K_{t}$, the next step is to actually measure the process to obtain $Y_{t}(27)$, and then to generate an a posterior state estimate by incorporating the measurement as in (25), the final step is to obtain an a posterior error covariance estimate via (26). After each time and measurement update pair, the process is repeated with the previous a posterior estimate used to project or predicts the new a priori estimates.

\section{Simulation Studies}

The largest Lyaponov exponent contains information on how far in the future predictions are possible. The Lyaponov spectrum has proven to be one of the most useful dynamic invariants that characterize chaotic dynamic systems. The Lyaponov exponents provide us with a measure of the averaged exponential rates of divergence or convergence of neighbor orbits in phase space [8]. If at least one positive Lyaponov exponent exists, the dynamic system is said to be chaotic, and the initial small differences between two trajectories will diverge exponentially.

We analyze the chaotic logistic map which has been hypothesized in a number of empirical studies of financial time series (see [9]). SNN is used with the coupled learning algorithm described in section 2 and the BNKF. After training, the networks are used in an iterative mode in order to generate a series of estimate outputs, and the dynamic invariants measured for the original series. The data set is displayed in figure 2. In this study the chosen model has parameters $d=2$ and $p=2, q=15$ (number of hidden units) respectively, that is:

$$
Y_{t}=h\left(\sum_{i=1}^{2} \varphi_{i}\left(\mathbf{X}_{t}\right) Y_{t-i}\right)+\xi_{t}
$$

Where $\mathbf{X}_{t}$ is a function of $\left\{Y_{t-1}, Y t_{-2}\right\}$ which are the neural network's inputs. The data set from 1 to 500 is used to train the neural network and from 500 to 1000 to test the neural network performance (figure2).

The network, during the training, learns the correct value of the Largest Lyaponov exponent that is 0.52 . Our analysis permits us to claim that the SNN capture "chaos" because it learns the dynamic invariant of a chaotic dynamic system. The simulated SNN and BNKF output are compared with the measured output in figure 3. As can be seen, the agreements are very good. 

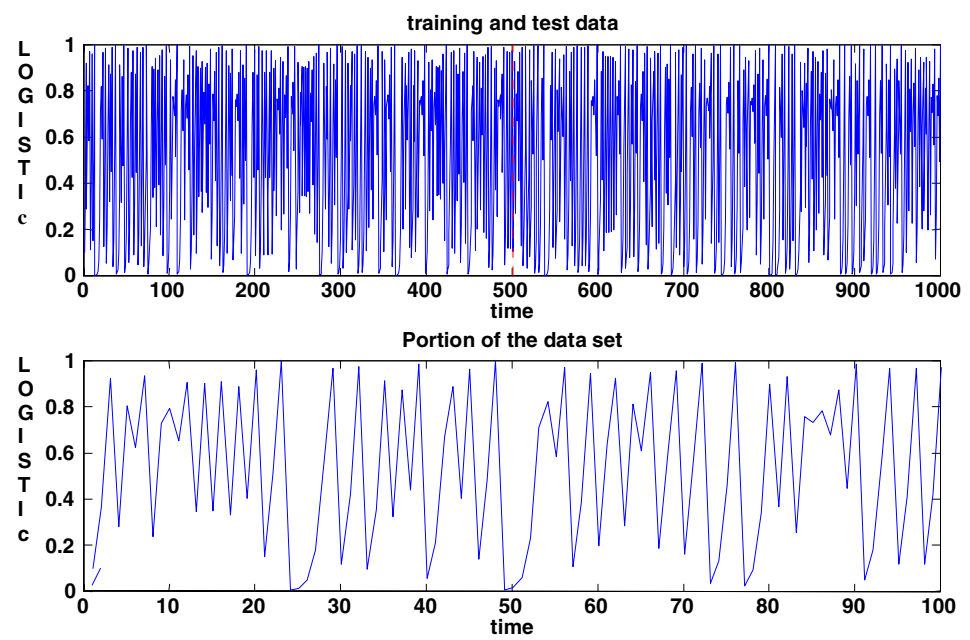

Fig. 2. . Data set from the Logistic map.
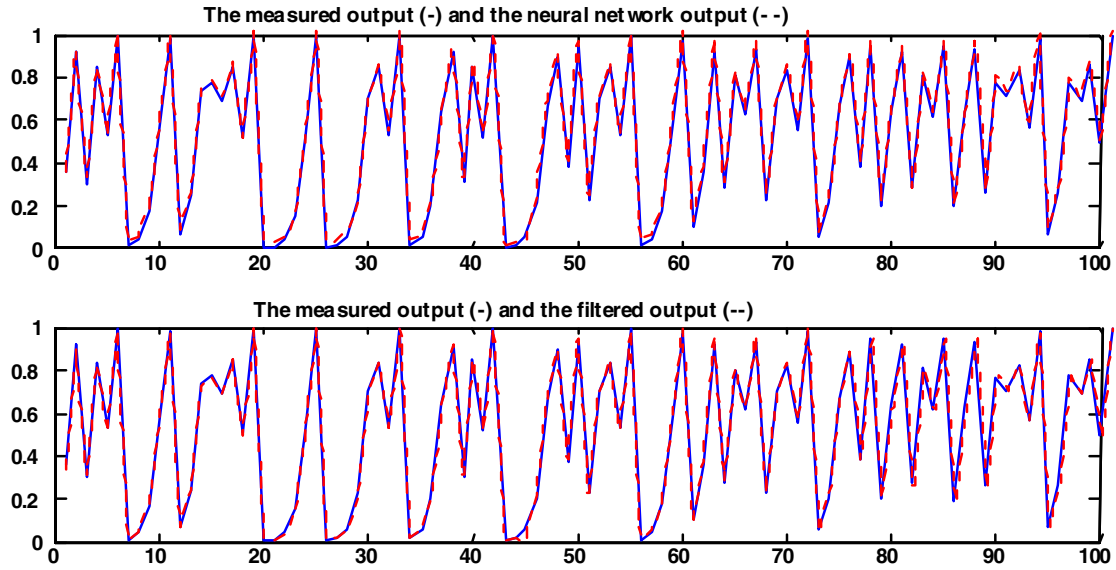

Fig. 3. Comparison of measured output and neural network model and filtered output.

\section{Analysis of (BVMT)}

To illustrate the application potential of the new SNN architecture on real data set, the case study, in this section, involved the modeling of the Tunisian Stock price index BVMT. The data set were daily closed values from Sept. 30, 1990 to Jul. 07, 1998. Figure 4 shows a temporal plot of the Tunisian Stock price index 


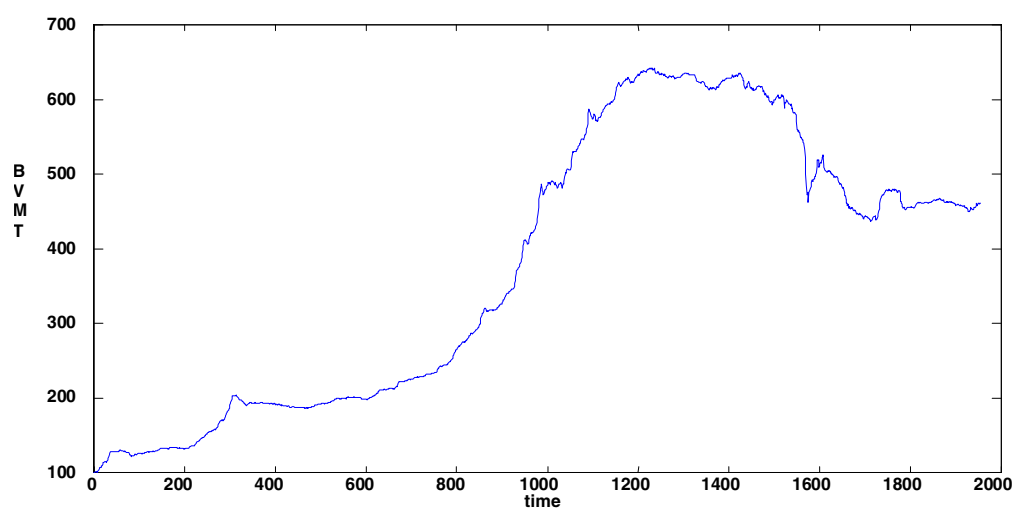

Fig. 4. Tunisian Stock Price Index (BVMT).

As we can see, the movements of the index not follow a random walk, and the system dynamics are not smooth, then the linear approximation of this dynamics is not appropriate. Identification of this non-linear structure by the SNN can clearly enable formulation of such system.

The variables of interest in this study are:

1. BVMT: Tunisian stock Price Index.

2. PE : Price/Earning ratio for Tunisian Stock Price.

These particular set of explanatory variables were selected because they are the types of variables used heavily by professional investors. The first Lag BVMT $\left(\mathbf{B V M T}_{t-1}\right.$ ) was also used as input for the SNN. The BVMT, $\mathbf{B V M T}_{t-1}$ and the PE were transformed by the first difference operator (D). The time series that can be modeled by the $\mathbf{S N N}$ is:

$$
\mathbf{B V M T}_{t}=f\left(\mathbf{B V M T}_{t-l}, \mathbf{P E}_{t}, \varphi\left(\mathbf{B V M T}_{t-l}, \mathbf{P E}_{t}\right), \gamma\left(\mathbf{B V M T}_{t-l}, \mathbf{P E}_{t}\right)\right)+\xi_{t}
$$

The network architecture of the SNN was determined in part by the domain variables. Since tree variables were selected as input stream to provide a univariate forecast. A common difficulty in applying neural networks lies in over fitting the data. A rule of thumb in the field of statistical modeling specifies that, for a case base of $\mathrm{N}$ observations, the degrees of freedom in the model should not exceed $\mathrm{N}^{0.5}$. Since our training set is fixed at 1000 observations, an upper bound would be about 32 weights, each corresponding to a degree of freedom in the SNN to be trained. Initially, the configuration $3 * 8 * 1=$ (corresponding to $3 * 8+8=32$ weights) was evaluated. However, the $3 * 7 * 1$ architecture yielded slightly better performance in term of prediction error. In this paper, the value of 7 hidden units was chosen for the $\mathbf{S N N}$ first layer. The $\mathbf{S N N}$ was trained and validate by the gradient back propagation and the BNKF described in section 3 and 4 (for $\mu=0.1$ ).

The performance among the predictive model is presented in figure5. The SNN output is closed to the desired output. Figure6 shows the evolution of the estimate Lyaponov exponent as function of the number of iterations via the SNN. It is important to remark that no overtraining is observed, the largest Lyaponov exponent is 1,48 . We conclude that the BVMT index is chaotic and stochastic. 


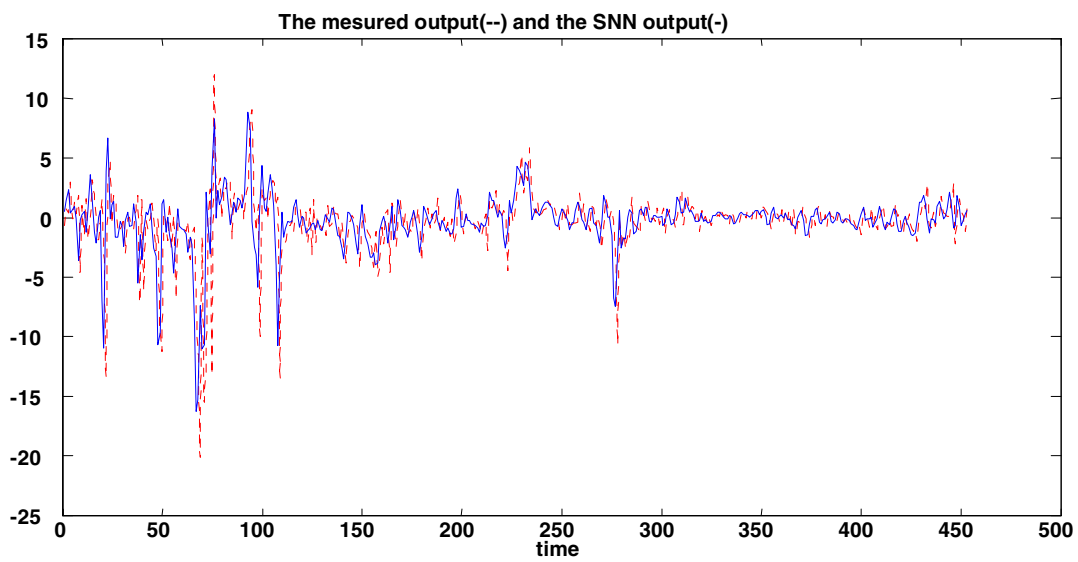

Fig. 5. Comparison of measured output and SNN output

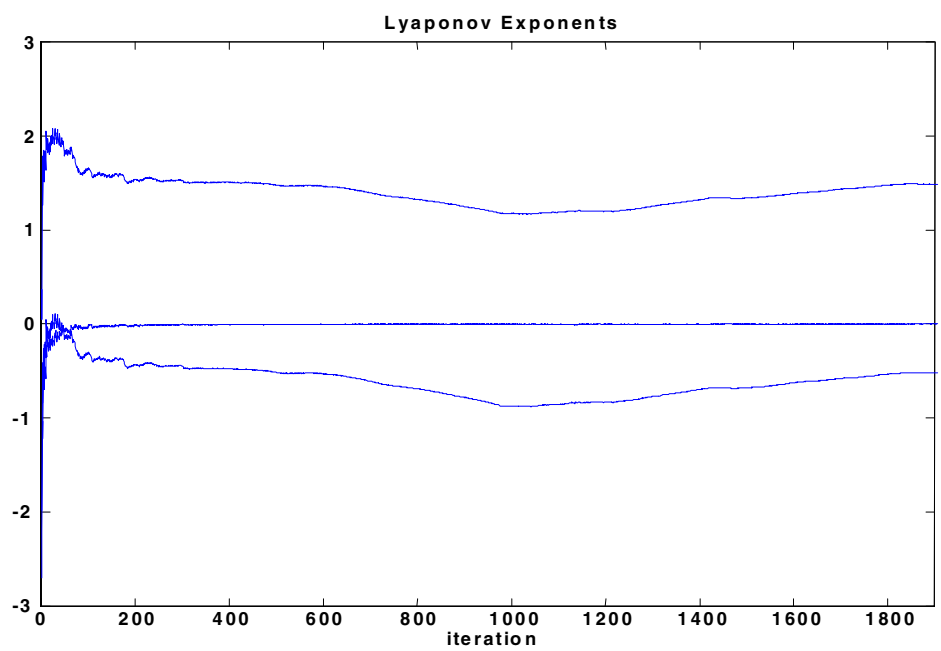

Fig. 6. Lyaponov exponents as a function of the number of iteration for the SNN trained by the BNKF of the BVMT.

\section{Conclusion}

This paper proposed a new approach based on Extended Kalman filter to help diagnose the dynamic structure of non linear time series.

For a set of input and output, a SNN can be used to identify the nonlinear time series of (1). A non linear state space form for this net is presented and a Bayesian algorithm for this model is derived to estimate the state.

Our analysis permits us to claim that the SNN capture "chaos" because it learns the dynamic invariants of a chaotic dynamic system (Logistic map). 
The proposed model was applied to the Tunisian stock index (BVMT) index). Our findings indicate that the BVMT index is chaotic and stochastic; the observed largest Lyaponov exponent is 1.48682 .

We believe that the encouraging results obtained herein with respect to the neural modeling of chaotic systems, in combination with existing linear and nonlinear dynamic techniques [10], [11] and [12], has a great potential for the modeling of financial, economic and other time series generated by complex market driven systems.

\section{References}

1. Blank, S.C.: Chaos in future Markets? a Nonlinear Dynamical Analysis. The Journal of Future Markets. 11.6 (1991) 711-728

2. Albano, A., Passamante, A., Hediger, T., Farell, M. E.: Using Neural Nets to look for chaos. Physica D. 58 (1992) 1

3. Nychka, D., Ellner, S., Gallant, A.R., Maccaffrey, D.: Findings chaos in noisy systems. Journal of the Royal Statistical Society, Series B. 54 (1992) 399-426

4. Hertz, J., Krogh, A., Palmer, R.: Introduction to the Theory of Neural Computing, Santa Fe Institute Studies in Science of Complexity, Amsterdam: Addison-Wesly (1991)

5. Weigend, .A.S., Huberman, B.A., Rumelhart, D.E.: Predicting the Future: A Connectionnist Approach. International Journal of Neural Systems, Vol 1.3 (1991) 193-209

6. Hornik, K., Stinchcombe, M., White: Multilayer feed forward networks are universal approximators. Neural Networks, vol.2 (1989) 359-366.

7. Aoki, M.: State-Space Modeling of Times Series, Berlin: Springer-Verlag (1987)

8. Wolf, A., Swift, J.B., Swinney, H.L., Vastano, J.A.: Determining Lyaponov Exponents from a time series. Physica D. 16 (1985) 285-317

9. Hsieh, D.A.: Chaos and Nonlinear Dynamics:Application to Financial Markets. The Journal of Finance. 6.5 (1991) 1839-1877

10. Hillmer,S.C, Trabelsi, A.: Benchmarking of Economic Time Series. Journal of the American Statistical Association: Theory and Methods. 82 (1987) 1064-1071

11. Trabelsi, A., Hillmer, S.C.: A Benchmarking Approach to Forecast Combination. Journal of Business and Economic Statistics. (1989) 353-362

12. Trabelsi, A., Hillmer, S.C.: Benchmarking Time Series with Reliable Benchmarks. Journal of the Royal Statistical Society, Applied Statistics. 39:3 (1990) 367-379. 